\begin{abstract}
¿Cómo citar este artículo?
García, A., y Fernández, I. (septiembre-diciembre, 2020). Auto-ocultación del acoso escolar si se es victima, agresor o testigo, y su vinculación con el bienestar subjetivo. Revista Virtual Universidad Católica del Norte, (61), 150-165 https://www.doi.org/10.35575/rvucn.n61a9
\end{abstract}

\title{
| Auto-ocultación del acoso escolar si se es víctima, agresor o testigo, y su vinculación con el bienestar subjetivo
}

Self-concealment of bullying, if student is victim, aggressor or witness, and their relationship with subjective well-being

\author{
Andrés García \\ Doctorando de Psicología Social \\ Facultad de Psicología, Universidad Nacional \\ de Educación a Distancia -UNED- \\ Madrid, España \\ andrexgg@gmail.com \\ Orcid: https://orcid.org/0000-0001-9199-4490
}

\author{
Itziar Fernández \\ Doctora en Psicología Social \\ Facultad de Psicología, Universidad Nacional \\ de Educación a Distancia -UNED- \\ Madrid, España \\ ifernandez@psi.uned.es \\ Orcid: https://orcid.org/0000-0002-6905-2111
}

Recibido: 20 de mayo de 2020

Evaluado: 16 de julio de 2020

Aprobado: 25 de agosto de 2020

Tipo de artículo: Investigación Científica y Tecnológica.

\section{Resumen}

El bullying es un comportamiento muy frecuente y ocurre sin ninguna provocación aparente por parte de quien lo sufre. Con el objeto de analizar cómo influye la auto-ocultación sobre el bienestar subjetivo, cuando se está implicado en situaciones de acoso escolar, se realizó una investigación con una muestra de 299 estudiantes, en centros de Lanzarote (Canarias, España), a través de un diseño correlacional donde cada alumno se auto-identifica como víctima, agresor o testigo. Los participantes tenían una media de 11.81 años (DT=.87), y el 50,2 \% eran hombres. Los resultados descriptivos mostraron diferencias significativas respecto a las víctimas, consistentes en menor bienestar subjetivo y mayor auto-ocultación. La auto-ocultación del bullying, frecuencia de acoso escolar y edad, explicaron el 29,1 \% de la varianza del bienestar subjetivo de los alumnos. El análisis de mediación moderada constató que a más frecuencia de acoso escolar se produce una mayor auto-ocultación, y que, a su vez, esta mayor auto-ocultación se asoció negativamente al bienestar subjetivo, tanto para las víctimas como para los agresores. En conclusión, los resultados de este trabajo confirman que una elevada auto-ocultación, cuando sucede el acoso, es perjudicial para el bienestar subjetivo.

Palabras clave: Acoso escolar; Auto-ocultación; Agresor; Bienestar subjetivo; Testigo; Víctima. 


\section{Abstract}

Bullying is a very frequent behavior and it occurs without any apparent provocation on the part of the victim. This research analyzed how self-concealment influences subjective well-being when involved in bullying situations, with a sample of 299 participants, in educational centers in Lanzarote (Canary Islands, Spain). The study was carried out with a correlational design. Each student self-identifies as victim, aggressor or witness. The participants had a mean of 11.81 years $(S D=.87), 50,2 \%$ were boys. The descriptive results showed significant differences, so the victims had lower subjective well-being and higher self-concealment. The self-concealment of bullying, frequency of bullying and age explained $29,1 \%$ of the variance of the students' subjective well-being. The moderate mediation analysis found that the more frequency of bullying higher self-concealment. This greater self-concealment was negatively associated with subjective well-being for both victims and aggressors. In conclusion: the results confirm that high self-concealment, when bullying occurs, is detrimental to subjective well-being.

Keywords: Bullying; Self-concealment; Aggressor; Subjective well-being; Witness; Victim.

\section{| Introducción}

El acoso escolar, que se produce de manera constante y reiterada en los centros educativos, tanto públicos como privados, es un hecho que preocupa a toda la sociedad. Este comportamiento violento es difícil de cuantificar, ya que se ejerce y mantiene, la mayoría de las veces, de manera oculta. Es más, la revisión realizada en 40 países sobre la prevalencia del bullying y la victimización, entre los niños de 11, 13 y 15 años, con muestras representativas y constituidas por un total de 202.056 jóvenes, informa que el rango varía del 4,8 \% al 45,2 \%; los niveles más elevados pertenecen a los países bálticos (Lituania, Letonia y Estonia), y los más bajos a los del norte de Europa. España, aunque está en el sur de dicho continente, ocupa el tercer lugar, tras Suecia y la República Checa (Craig et al., 2009).

Este rango de más de 40 puntos se reduce, según la estimación de Rettew \& Pawlowski (2016), aunque igualmente sigue siendo muy preocupante. Para estos autores entre un $10 \%$ y un $30 \%$ de los escolares en todo el mundo están involucrados en episodios de bullying.

Datos más recientes constatan un total de 1.054 casos denunciados en España en 2017 (Instituto Nacional de Estadística -INE-, 2019). La franja de edad en donde el acoso presenta un mayor porcentaje, o cuando los implicados se atreven a verbalizarlo, es la comprendida entre los 12 y 14 años, con 459 denuncias, seguida de los 15 a 17 años, con 352 denuncias. A distancia estaría la de los más pequeños ( 6 a 11 años), de acuerdo con las estadísticas oficiales ofrecidas por las fuerzas de seguridad (Ministerio de Educación, Cultura y Deporte-MECD-, 2017).

En general, el acoso escolar o Bullying son conceptos para referirse al maltrato, hostigamiento e intimidación de manera deliberada y continuada que ejerce un niño o grupo hacia otro de forma cruel, mediante agresiones verbales (insultos, ofensas), físicas (empujones, golpes), aislamiento (invisibilidad, ninguneo, ostracismo, exclusión social), difusión de rumores (descrédito, bulos), robo o rotura de material escolar y ciberbullying (Garaigordobil et al., 2017; Menesisi \& Salmivalli, 2017).

Dan Olweus (1993, 2003, 2011, 2013), pionero en el estudio de esta problemática, lo describe como una conducta agresiva sistemática y sin motivo hacia alguien, con indefensión por parte de la víctima, realizada de manera persistente en el tiempo y donde se da un desequilibrio de poder. En esta definición es importante considerar el matiz de persistencia, que le confiere el carácter de acoso y lo diferencia de la agresión puntual. 
El aula, los patios de recreo, los pasillos, los aseos y los alrededores del centro educativo son escenarios habituales de episodios violentos en los que hay víctimas, agresores y testigos. Según el estudio realizado por la Fundación Ayuda a Niños y Adolescentes en Riesgo -ANAR-(2018) lo más frecuente es que los agresores pertenezcan a la misma aula de las víctimas (88,1 \%), teniendo un 10,2 \% de ellos más edad que las víctimas y, por consiguiente, de clases diferentes. La conducta caracterizada por la agresividad verbal (por ejemplo, burlas) es la más habitual entre los agresores (78,0 \%), mientras que los ataques físicos (por ejemplo, patadas) se producen la mitad de las veces (51,6 \%). El acoso escolar suele perdurar más de un año para el 52,9\% de las víctimas, y para una tercera parte (37,6\%) el tiempo está comprendido entre un mes y un año.

Un porcentaje muy elevado de las víctimas (94\%), según ANAR (2018), presenta secuelas psicológicas tras sufrir bullying. Por orden de importancia destacan la sintomatología depresiva $(68,8 \%)$, los ataques de ansiedad (67,2\%) y la emoción de miedo (58,6 \%). También, se pueden producir aislamiento (26,3\%), sentimientos de soledad (23,7\%), falta de competencias sociales (19,4\%), bajo rendimiento académico (16,7 \%), problemas de autoestima (15,6 \%) y la emoción de enfado (15,6 \%). En esta línea, la investigación de Lenci \& Matuga (2010) ha constatado la relación entre el acoso escolar y un mayor riesgo de depresión.

En lo que respecta a las características de las víctimas, la revisión realizada por Puértolas y Montiel (2017) constató que una gran parte de quienes sufren acoso escolar presentan baja autoestima, baja asertividad, timidez y otra sintomatología internalizante, aunque también ocasionalmente externalizante; asi como revictimización frecuente a lo largo de su escolarización. Además, muestran de manera habitual carencias en habilidades y relaciones sociales, con sentimientos de rechazo y aislamiento; son poco populares y poseen un círculo de amistades muy reducido.

Más allá de todas estas cifras y características, es una conducta que resulta muchas veces difícil de ser prevenida, y que al ejercerse habitualmente de forma oculta hace que exista una apariencia de normalidad entre todos los implicados en este proceso. Entre ellos se destacan los agresores, quienes infringen el menosprecio a la víctima; los testigos, que son aquellos que observan lo que ocurre; y la víctima, o persona que sufre el acoso. Se trata de un triángulo que puede acoger a más participantes. Así, Saarento \& Salmivalli (2015) identifican otros roles, a saber: a) los defensores, que se sienten seguros e intervienen para interponerse ante el acoso, y b) los agresores-víctimas (que acosan a otros, pero a su vez son víctimas de bullying). Estos suelen ser impulsivos y cuando son acosados reaccionan violentamente, a diferencia de las victimas sin el componente ofensivo, que se caracterizan por su inseguridad, inhibición, vulnerabilidad y miedo hacia sus agresores.

Estas clasificaciones han sido matizadas por otros autores. Por ejemplo, Millán Reyes et al. (2015) diferencian a los espectadores, como testigos activos y pasivos. Los primeros tratan de evitar que se agreda a las víctimas e intervienen para intentar frenar los incidentes de bullying, mientras que los segundos optan por permanecer quietos e impasibles cuando se produce el acoso hacía otro compañero; son los denominados cómplices del suceso. Más recientemente, Conde y Avila (2018) analizan el papel de otro tipo de observadores, los denominados reforzadores, los cuales aprueban la violencia del agresor hacia su víctima, con risas. 


\section{Auto-ocultación y acoso escolar}

En la dinámica de acoso, este triángulo que puede expandirse hacia otros integrantes, como se termina de describir, está caracterizado por su asimetría o desequilibrio de poder; aspecto clave dentro del bullying, como se veía anteriormente en la definición clásica de Olweus. Esta asimetría entre víctima y victimario opera también en la puesta en práctica de diversos mecanismos de ocultación de dicha acción y sus consecuencias. Eso explica que muchos acosos escolares nunca lleguen a conocerse, debido a que las víctimas intentan de ese modo escapar a consecuencias mucho más graves. El silencio forzado de las víctimas encontraría aquí su explicación (Quiles del Castillo et al., 2014).

Darley (1996), por su parte, analiza los costes de estas ocultaciones para los agresores: a saber: i) es el peligro siempre presente de que resulten contraproducentes, porque la evidencia está ahí es clara, puede haber testigos, y evitar su difusión a través de filtraciones es complicado; ii) el hecho mismo de la ocultación proporciona evidencia concluyente del carácter malicioso de las acciones; y iii) la ocultación es, habitualmente, resultado de una fuerte presión, sobre todo para mantener la propia imagen del yo o protegerse ante la situación.

También, hay beneficios para los que renunciaron a denunciar el acoso; es decir, para los testigos. Su connivencia con el suceso, su tolerancia de las prácticas de acoso, acaba por consolidar el sistema y le otorga un carácter de perennidad. Ahora estas prácticas parecen naturales, como si siempre hubieran estado ahí; por tanto, la relación trilateral se sostiene mediante la "Ley del silencio" y la condena pública del delator o "chivato". En este sentido, Salmivalli (2013) indica que:

Los escolares que afrontan el bullying como testigos se encuentran atrapados en un dilema social. Por un lado, comprenden que el acoso está mal y les gustaría hacer algo para pararlo o evitarlo, pero, por otro lado, deben esforzarse por asegurar su estatus y seguridad dentro de su grupo de iguales. (p. 122)

Como se ha señalado, la ocultación está muy relacionada con el acoso; es por ello que el presente estudio se centra en la auto-ocultación, la cual es definida como una predisposición a ocultar activamente, a los demás, información personal que uno percibe como angustiante o negativa. Esta información personal, oculta de pensamientos, sentimientos, acciones o eventos es muy íntima, de valencia negativa y tiene tres características: i) es un subconjunto de información privada, ii) a esta información se puede acceder conscientemente, y iii) dicha información se esconde activamente a los demás (Larson et al., 2015). Esta conducta contribuye negativamente sobre la salud psicológica. Así, la auto-ocultación personal fue un mediador significativo entre los episodios de discriminación y la ansiedad (Zhou et al., 2019).

Por su parte, Bustillos et al. (2008), en un estudio sobre variables psicosociales y acoso escolar, llegan a la conclusión de que la exclusión social es predictora del inicio de situaciones de acoso escolar. Es decir, puede ocurrir que, por las características internalizantes, que se consideran habituales en un tipo concreto de víctimas, como por el hecho de mostrar una mayor propensión a la timidez, soledad, miedos, fobias, etc., tiendan por sí mismos a mantener su actividad social al mínimo, lo que hace que acaben siendo excluidos socialmente. Todo esto prepara, según los autores, el terreno para que puedan iniciarse comportamientos de acoso escolar. Aunque también puede suceder que lo llamativo sean las características externalizantes y disruptivas en el grupo, como el alumnado con sintomatología relacionada con el TDAH (Trastorno por Déficit de Atención e Hiperactividad), lo que propicie la exclusión, pues las víctimas no suelen acatar las normas del grupo, se despistan más y cometen más torpezas en los juegos. 
En cualquiera de los casos, bien por factores internos o externos, se podría decir que un indicador previo al inicio del acoso es la existencia de cierto grado de exclusión social. Esto encaja con la posible existencia de una mayor auto-ocultación, pues parece que los niños y niñas que sufren acoso escolar son especialmente susceptibles a tener tasas más altas de ocultación, generalizada a todos los ámbitos de su vida familiar, escolar y comunitaria, por lo que es muy probable que, una vez iniciado el acoso escolar, no se detenga hasta llegar a manifestaciones que ya no son admisibles grupalmente.

\section{Bullying y bienestar subjetivo}

En este estudio, además, se analiza cómo sufrir acoso, infringir agresiones de manera continuada o ser testigo, inciden sobre el bienestar subjetivo de los escolares. En este sentido, el informe de la Organización Mundial de la Salud sobre el bienestar de los niños constató que el clima escolar y la calidad de las relaciones entre los niños son los factores más importantes para predecir el bienestar subjetivo (Klocke et al., 2014). Así, la investigación realizada por Navarro et al. (2015) concluyó que existe una relación entre el bullying y el poseer un menor optimismo y satisfacción con los amigos; el acoso escolar reduce de forma significativa la sensación de felicidad y la satisfacción. Otros estudios empíricos confirmaron que el auto-ocultamiento se asocia negativamente con el bienestar subjetivo (Uysal et al., 2012), y que la ocultación personal inhibe el bienestar psicológico (Wang et al., 2014).

El indicador de la ocultación ha dado origen a un gran número de investigaciones en ámbitos como el maltrato infantil y la violencia de género (Bello, 2014), pero no hay muchos estudios que aborden la auto-ocultación y el bullying.

Por todo lo anteriormente expuesto, el objetivo de la presente investigación es evaluar cómo opera la auto-ocultación, tras el inicio de conductas de acoso escolar, en función del papel que tiene cada participante (víctima, agresor o testigo).

Para llevar a cabo el estudio se contó con un diseño correlacional, donde los alumnos contestaron, a través de un autoinforme, a una serie de cuestiones que guardan relación con las siguientes hipótesis:

H1. Los participantes que son víctimas y agresores puntuarán más en la auto-ocultación del acoso escolar, que los testigos de la situación.

H2. Las víctimas de bullying mostrarán peor bienestar subjetivo que los agresores y testigos.

H3. Los participantes con un nivel bajo de auto-ocultación de bullying presentarán un mayor bienestar subjetivo, que los participantes con un nivel alto de auto-ocultación del acoso escolar.

H4. Las víctimas que auto-ocultan más el acoso escolar mostrarán menor bienestar subjetivo, que las víctimas que no lo auto-ocultan.

H5. Los participantes que sufren una alta frecuencia de acoso escolar auto-ocultarán más el bullying, lo que a su vez se asociará con un menor bienestar subjetivo. Sin embargo, este proceso mediador sólo se manifestará entre las víctimas y los agresores (véase figura 1). 


\section{Figura 1}

Modelo de mediación moderada

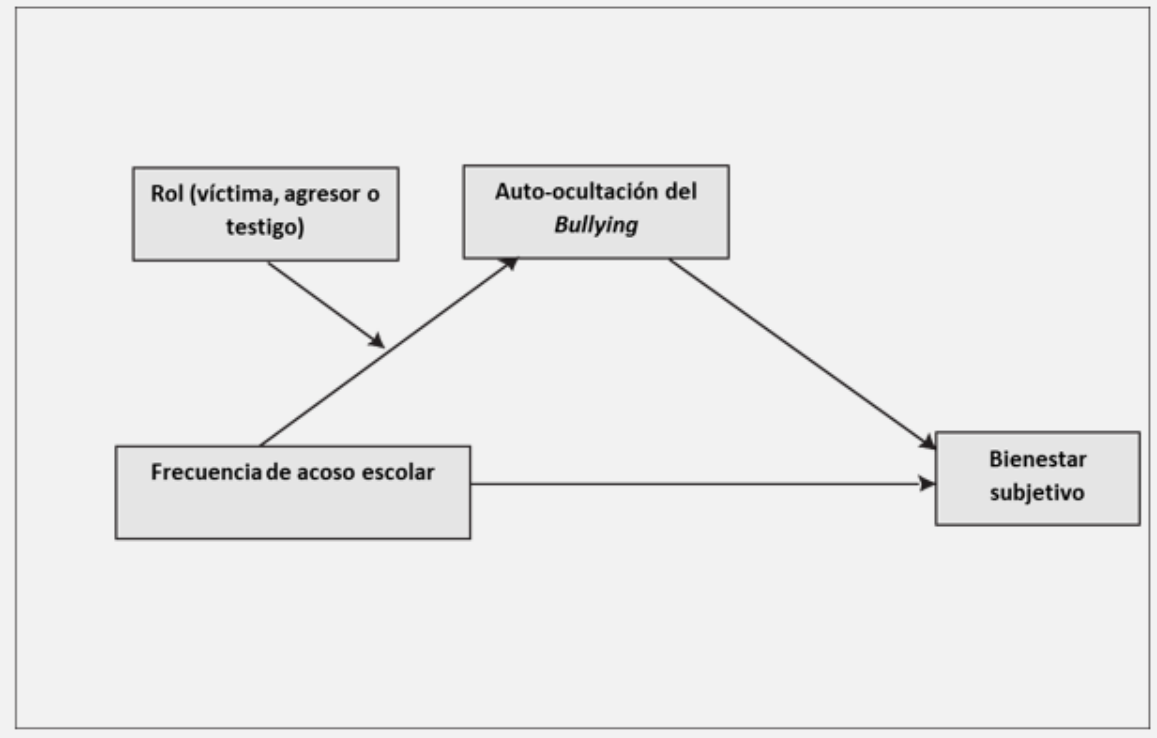

Nota: Elaboración propia

\section{| Metodología}

\section{Procedimiento}

Se recogieron las respuestas de todos los participantes en cinco centros educativos pertenecientes a la Isla de Lanzarote (España). La elección se basó en el criterio de accesibilidad, si bien se trató de contar con un número representativo de estudiantes pertenecientes a distintos colegios. El cuestionario contenía varias escalas (véase el epígrafe de instrumentos y variables) y se aplicó de forma colectiva en las aulas de cada uno de los centros, por un único asistente de investigación. Antes de comenzar con la batería de preguntas se solicitó el consentimiento informado, tanto a los alumnos como a sus tutores legales. Todos conocían el propósito del estudio y que la colaboración en el mismo no conlleva ningún riesgo para la salud. La participación en el estudio fue voluntaria, garantizándose el anonimato y confidencialidad de las respuestas. 


\section{Muestra}

Para la presente investigación se contó con 299 participantes. El 50,2 \% ( $n=150)$ eran hombres, con una edad media de 11.81 (DT=.87), y rango de variación de 10 a 13 años; además, los estudiantes cursaban sexto de primaria y primero de secundaria, y se auto-identificaron como víctimas, agresores y testigos. Así, el grupo de víctimas, según su percepción, lo constituían estudiantes con una media de 11.72 años $(D T=.87)$, siendo 19 hombres y 27 mujeres. El conjunto de alumnos que decía realizar conductas de bullying presentó una media de 12.19 años (DT=1.03), y estuvo formado por 8 hombres y 5 mujeres. Por último, el grupo de testigos, que indicó presenciar y/o conocer conductas de acoso escolar, presentaba una edad de 11.8 años (DT=.86), donde 123 eran hombres y 117 mujeres.

\section{Instrumentos y variables}

Acoso escolar. Para evaluar el acoso escolar se tuvieron en cuenta dos aspectos, la presencia del bullying y el rol de cada participante. Por una parte, la presencia se midió a través de una pregunta extraída de la encuesta realizada por el Defensor del Pueblo (2007) donde el estudiante tenía que responder acerca de la frecuencia con que había sufrido conductas de acoso durante el presente curso académico. Se evaluaron comportamientos como ser pegado, empujado, gritado, insultado, amedrentado, robado, excluido o ignorado. Se utilizó una escala con cuatro opciones de respuesta, tipo Likert, de 1=Nunca a 4=Siempre. Los porcentajes fueron los siguientes: nunca ( $65,8 \%$ hombres y $58,1 \%$ mujeres), a veces (23,5 \% hombres y $22 \%$ mujeres), a menudo ( $9,4 \%$ hombres y $18,2 \%$ mujeres), y siempre (1,3\% hombres y 1,7 \% mujeres). Por otra parte, se examinó qué papel desempeñaba cada uno de los alumnos ante estas conductas de acoso; el mayor porcentaje fue para el rol de testigo, con 240 participantes (80,3 \%), seguido del de víctima $(15,4 \%, n=46)$, y por último los agresores con $13(4,3 \%)$.

Auto-ocultación. Self-Concealment Scale-SCS- (Larson \& Chastain, 1990). La escala fue traducida por el primer autor de este artículo, adaptando algunas expresiones al objeto de estudio (Finkenauer et al., 2002). Se trata de una medida que evalúa la tendencia a ocultar información personal angustiante o evaluada de forma negativa sobre cualquier problema o suceso vital estresante (por ejemplo, "Algunos de mis secretos realmente me angustian"). El instrumento contiene 10 preguntas, donde 1 es "totalmente en desacuerdo" y 5 es "totalmente de acuerdo", siendo su rango de variación de 10 a 50, de manera que cuando la puntuación es elevada la auto-ocultación es mayor. Los estadísticos de tendencia central fueron $(M=22.73$; $D T=9.79$ ). El nivel de auto-ocultación bajo vs. alto se operativizó en función de la mediana (valor 21), con el objeto de contar con un criterio estadístico que permitiera realizar una comparación válida. Hubo diferencias significativas para la variable sexo $\left(F(1,297)=5.12,<.05, M_{\text {mujeres }}=23.66\right.$; $D T=10.11$ vs. Mhombres=21.82; DT=9.39). El índice de fiabilidad, el alfa de Cronbach fue satisfactorio: .87.

Bienestar subjetivo. Se aplicó la pregunta de Diener et al. (1985) ¿Hasta qué punto estás actualmente satisfecho con tu vida, considerada globalmente? con un rango de variación entre 1 (totalmente insatisfecho) y 10 (totalmente satisfecho) (Adaptada al contexto cultural por Páez et al., 2011). La medida es subjetiva, ya que se evalúa la experiencia de la persona y el bienestar es global, pues incluye una valoración o juicio general de su vida (Diener et al., 1999). El conjunto de los participantes mostró una puntuación alta $(M=8.39 ; D T=1.57)$, y hubo diferencias significativas para la variable sexo $(F(1,295)=5.15,<.05)$. $M$ mujeres $=8.24 ; D T=1.67$ vs Mhombres $=8.53 ; D T=1.45$.

Datos sociodemográficos. Tras la aplicación de estas medidas se preguntaba por cuestiones referidas al sexo y la edad. 


\section{Análisis estadísticos}

Se utilizó el programa SPSS (versión 24). La fiabilidad de las medidas se comprobó a través del alfa de Cronbach $(\alpha)$. Para el nivel de significación se ha considerado una probabilidad de error menor o igual al 5\% ( $p<.05)$. La comparación de medias se ha realizado a través de los análisis de varianza, con su respectivo tamaño del efecto (eta cuadrado parcial $\eta p^{2}$ ). Las relaciones para el conjunto de variables objeto de estudio se han calculado a través de las correlaciones $r$ de Pearson, de la regresión lineal y del análisis de mediación (macro PROCESS 3.0; Hayes, 2018).

\section{Resultados}

\section{Análisis previos}

Con el objeto de comprobar que los participantes pertenecientes a los cinco centros educativos estaban equiparados con respecto a la frecuencia de haber sufrido bullying y su rol de víctima, agresor o testigo, se realizaron análisis de varianza (ANOVA) en los que se incluyó, como variable independiente (VI), la pertenencia a cada centro. Como variables dependientes (VDs) los dos ítems descritos anteriormente (véase la variable acoso escolar). Los resultados mostraron que no existen diferencias estadísticamente significativas (ambas Fs $(4,294), n s)$. El nivel de significación se estableció con una $p>$.01. Este resultado permite confirmar la homogeneidad de los distintos centros, respecto al acoso escolar.

\section{Quien oculta más el acoso escolar}

Para constatar la primera hipótesis se efectuó un análisis univariado; el papel de cada alumno ante el acoso (víctima, agresor o testigo) como VI y la auto-ocultación como VD. Los resultados confirmaron que las víctimas y agresores auto-ocultan más el bullying que los testigos (Mvictimas=30.31, DT=10.71, Magresores=26.05, $D T=10.21$ y Mtestigos=21.30, DT=8.98), $F(2,294)=33.99, p$ $\left.<001, n p^{2}=.11\right)$. Una vez determinada esta diferencia de medias, se realizaron comparaciones múltiples post hoc a través de la prueba T3 de Dunnett; este análisis permitió determinar que la mayor diferencia de auto-ocultación de acoso escolar se da entre las víctimas y los que observan los episodios de bullying $(M=9.01, E E M=1.26 ; p<001)$.

Además, y aunque no se tenía ninguna hipótesis de investigación, con el propósito de tener más información sobre quién auto-oculta más, si los hombres o las mujeres, o los que son más jóvenes o tienen más años, se realizó un ANOVA donde la VI fue la variable dummy, baja vs. alta auto-ocultación y la VD la edad. Los resultados indicaron que eran los participantes con más años los que auto-ocultaban más el acoso (Mbaja auto-ocultación=11.70, DT=.86 y Malta auto-ocultación $=11.89, D T=.87 ; F(1,294)=5.27 ; p<.01)$. Las diferencias de sexo se comprobaron a través de la prueba t-test para muestras independientes (Mmujeres=23.66, $D T=10.17$ vs. Mhombres $=21.82, D T=9.39 ;$ t-test $(297)=-2.26 ; p<.05)$, lo cual permitió confirmar que son las mujeres las que ocultan más el bullying. 


\section{Cómo se relaciona el bullying y el bienestar subjetivo}

La segunda hipótesis se comprobó, en primer lugar, mediante un análisis de correlación, donde se vinculó la frecuencia de acoso con el bienestar subjetivo $(r(294)=-.40, p<001)$; este resultado permitió determinar que a más acoco escolar menor bienestar subjetivo, o que a más bienestar subjetivo menor bullying. En segundo lugar, a través de un análisis univariado, se tuvo en cuenta como VI el papel de cada alumno ante el acoso (víctima, agresor o testigo) y como VD el bienestar subjetivo. Los resultados, como cabía esperar, confirmaron que los participantes que tenían el rol de testigos poseían un mayor bienestar, seguidos de los agresores y las víctimas

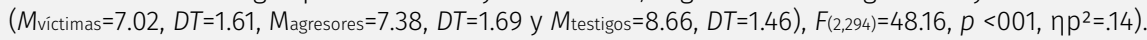
Respecto a las comparaciones múltiples entre estos tres grupos, la prueba T3 de Dunnett confirmó que existen diferencias entre los testigos y las víctimas $(M=1.64, E E M=.19 ; p<001)$ y entre los testigos y los agresores $(M=1.28, E E M=.37 ; p<01)$, no habiendo diferencias estadísticamente significativas para la comparación entre las víctimas y los agresores; esto hace pensar que ambos colectivos, tras infligir y sufrir el bullying, respectivamente, tienen un bienestar subjetivo similar (valor 7). Por tanto, la hipótesis se ve confirmada en parte, ya que las víctimas tienen peor bienestar subjetivo que los testigos, pero su percepción de bienestar es similar a la de sus agresores.

\section{Cómo afecta la auto-ocultación al bienestar subjetivo cuando ocurre el acoso escolar}

Para constatar la tercera hipótesis se efectuó un ANOVA, con el nivel de auto-ocultación (bajo vs. alto) como VI, y el bienestar subjetivo como VD. Los resultados confirmaron que sí existe un mayor bienestar subjetivo en el nivel bajo de auto-ocultación $(M=8.97 ; D T=1.17)$ que en el alto $(M=7.86 ; D T=1.70)(F(1,297)=80.94 ; p<.001)$.

La cuarta hipótesis se comprobó a través del mismo análisis que el efectuado para la H3, pero en este caso se realizaron ANOVAs diferentes en función del papel de cada alumno ante el acoso (víctima, agresor o testigo). Los resultados corroboraron que si existe un mayor bienestar subjetivo en el nivel bajo de auto-ocultación del bullying para los agresores (Mbaja auto-ocultación=8.25, DT=1.83 y Malta auto-ocultación $=6.85, D T=1.41 ; F(1,12)=3.93 ; p<.05)$ y testigos (Mbaja auto-ocultación=9.09, $D T=1.06$ y Malta auto-ocultación $=8.18, D T=1.61 ; F(1,239)=54.21 ; p<.001)$, pero no para las víctimas (Mbaja auto-ocultaciôn=7.38, DT=1.25 y Malta auto-ocultación $=6.94, D T=1.68 ; F(1,45)=.94 ; n s)$. Por tanto, sí se confirma la $\mathrm{H} 3$, pero no la $\mathrm{H} 4$.

\section{La influencia del sexo, la edad, la auto-ocultación del bullying y la frecuencia de acoso escolar sobre el bienestar subjetivo}

Con el objeto de analizar en su conjunto la influencia del sexo, edad, auto-ocultación y frecuencia de acoso escolar sobre el bienestar subjetivo, se realizó una regresión (método: enter). El sexo resulto no significativo $ß=-.33$; $t=-.111 ; p<n s, 95 \%$ IC $[-.34, .09]$, mientras que las variable auto-ocultación del bullying ( $B=-.33 ; t=-.8 .76 ; p<.001,95 \%$ IC $[-.06,-.04]$ ), frecuencia de acoso escolar ( $\beta=-.29 ; t=-.7 .68 ; p<.001,95 \% \mathrm{IC}[-.72,-.43]$ ) y edad $(\beta=-.16 ; t=-.4 .49 ; p<.001,95 \% \mathrm{IC}[-.41$, -.16]) explicaron el 29,6\% ( $\left.R^{2}=.296\right)$ de la varianza del bienestar subjetivo de los alumnos $(F(4,294)=60.20, p<001)$. Por tanto, los estudiantes más jóvenes, que auto-ocultan menos el bullying y presentan una menor frecuencia de acoso, se caracterizan por un mayor bienestar subjetivo. 


\section{Frecuencia del acoso: la mediación moderada de la auto-ocultación del bullying a través del rol de estudiante sobre el bienestar subjetivo}

La hipótesis 5 trataba de constatar la auto-ocultación del bullying como variable mediadora de la frecuencia del acoso escolar hacia el bienestar subjetivo, pero manteniendo el rol del alumno como variable moderadora. Para poner a prueba la H5 se aplicó la macro PROCESS de Hayes (2018), concretamente el modelo 7 de mediación moderada.

Este procedimiento permite calcular los efectos indirectos condicionales, es decir, el efecto de la VI (frecuencia de acoso escolar) sobre una VD (bienestar subjetivo), a través de una variable mediadora (auto-ocultación del bullying), para diferentes niveles de una variable moderadora (rol: testigo, víctima y agresor). En este estudio los efectos indirectos condicionales se calcularon utilizando el método de bootstrapping (o remuestreo). Los coeficientes se estimaron a través de 10.000 muestras bootstrap (Hayes, 2009).

\section{Tabla 1}

Análisis de mediación moderada

\begin{tabular}{|c|c|c|c|c|}
\hline $\begin{array}{c}\left(R^{2}=.13 ; F_{(3,292)}=27.54\right. \\
p<001)\end{array}$ & \multicolumn{4}{|c|}{ Modelo de variable mediadora (Auto-ocultación del bullying) } \\
\hline Variables predictoras & $B$ & $S E$ & $T$ & $p$ \\
\hline Constante & 22.74 & .44 & 51.94 & .001 \\
\hline Acoso Escolar (AE) & 3.35 & .64 & 5.25 & .001 \\
\hline Rol & 2.62 & .96 & 2.73 & .01 \\
\hline Interacción AE x Rol & -1.04 & .22 & -2.04 & .05 \\
\hline $\begin{array}{l}\left(R^{2}=.27 ; F_{(2,293)}=106.97,\right. \\
p<001)\end{array}$ & \multicolumn{4}{|c|}{ Modelo de variable dependiente (Bienestar subjetivo) } \\
\hline Variables predictoras & $B$ & $S E$ & $T$ & $p$ \\
\hline Constante & 9.66 & .15 & 64.77 & .001 \\
\hline $\mathrm{AE}$ efecto directo & -.48 & .14 & -3.01 & .081 \\
\hline Auto-ocultación & -.06 & .01 & -9.25 & .001 \\
\hline & \multicolumn{4}{|c|}{ Efectos indirectos condicionales } \\
\hline Mediador & Moderador & $B$ & $S E$ & Boot 95\% IC \\
\hline Auto-ocultación & Rol: víctima & -.30 & .16 & {$[-.66,-.24]$} \\
\hline Auto-ocultación & Rol: agresor & -.28 & .14 & {$[-.62,-.27]$} \\
\hline Auto-ocultación & Rol: testigo & -.24 & .13 & {$[-.50, .07]$} \\
\hline
\end{tabular}

Nota: Elaboración propia. En la tabla se incluyen los coeficientes $B$ de regresión no estandarizados. Intervalo de Confianza (IC) si contiene el valor 0 es no significativo (Hayes, 2018). 
Los resultados de los análisis, que se presentan en la tabla 1, constatan que a más frecuencia de acoso escolar se produce una mayor auto-ocultación del bullying $(B=3.35, p<.001)\left(R^{2}=.13\right.$; $F(3,294)=27.54, p<001)$, y que, a su vez, esta mayor auto-ocultación se asoció negativamente al bienestar subjetivo $(B=-.06, p<.001)\left(R^{2}=.27 ; F(2,293)=106.97, p<001\right)$. El efecto directo de la frecuencia del acoso escolar sobre el bienestar $(B=-.48, p=.08)$ confirma que a más acoso menor bienestar subjetivo. Además, este coeficiente es no significativo en el efecto indirecto $(B=3.35, p$ $<.001$ ), lo que confirma que se produce la mediación de la auto-ocultación.

Por su parte, la existencia de un efecto de interacción entre acoso escolar y el papel de cada participante tuvo la siguiente significación ( $B=-1.04, p=.05$ ), lo que implica que los efectos indirectos condicionales solo son válidos para víctimas $(B=-.30 ; 95 \%$ IC $[-.66,-.24])$ y agresores $(B=$ $-.28 ; 95 \%$ IC $[-.62,-.27])$. Estos resultados, en su conjunto, permiten concluir que la auto-ocultación sí media entre la frecuencia de acoso escolar y el bienestar subjetivo, mientras que los roles de víctima y agresor tienen efectos moderadores sobre la auto-ocultación del bullying.

\section{| Discusión}

El acoso escolar representa un problema complejo debido a la diversidad de factores involucrados. En este artículo se ha tratado de aportar algunos indicadores desde una perspectiva psicosocial, estableciendo relaciones entre la auto-ocultación del bullying y el bienestar subjetivo. Además, se ha analizado el papel como víctima, agresor o testigo que juega cada uno de los implicados en esta relación triangular de acoso escolar. Los resultados descriptivos constatan que las características del bullying, recordadas por los estudiantes, son similares a las de otras investigaciones epidemiológicas llevadas a cabo en España (Oñate y Piñuel, 2010). Respecto a los testigos no solamente constituyen el porcentaje mayoritario de participantes (80,3\%), sino que dependiendo de sus acciones a favor o en contra de víctimas y agresores, incentivan, refuerzan, rechazan o ignoran el bullying (Cuevas y Marmolejo, 2016).

La primera hipótesis referente a la auto-ocultación del acoso escolar, en función del rol que se desempeña, bien como víctima, agresor o testigo, se confirmó, ya que los que ocultan más el bullying son las víctimas y los agresores. Además, se constató que son las mujeres y los que tienen más años, el perfil socio-demográfico de participantes que auto-ocultan más. En cuanto al papel de testigos que auto-ocultan la situación, es posible, tal y como indican Peets et al. (2015), que perciban su inseguridad e incapacidad para defender a la víctima de bullying.

Otro indicador a tener en cuenta es el bienestar subjetivo, ya que décadas de investigación han concluido que uno de los principales factores que afecta al bienestar subjetivo es la calidad de las interacciones sociales de un individuo (Heintzelman \& Diener, 2018). Es por ello que la segunda hipótesis trató de analizar la vinculación entre acoso escolar y bienestar subjetivo. La información empírica ha permitido confirmar que la mayor frecuencia de acoso escolar se relaciona negativamente con el bienestar. La H2 se corroboró en parte, ya que no sólo las víctimas tienen peor bienestar subjetivo que los testigos, sino también los agresores. En general, los resultados del presente estudio van en consonancia con las conclusiones de la investigación de Navarro et al. (2015), sobre la vinculación entre el acoso escolar con la reducción del optimismo, felicidad y satisfacción entre compañeros, en el tema de víctimas y victimarios. En esta misma línea, la victimización por bullying tuvo un impacto directo sobre el bienestar y un efecto indirecto a través del clima escolar en una muestra de 1829 niños de 12 años, pertenecientes a contextos tan diferentes como Chile y Sudáfrica (Varela et al., 2020). 
Las hipótesis 3 y 4 trataron de comprobar cómo afecta la auto-ocultación del bullying al bienestar subjetivo. En la H3 se tomó en su conjunto a todos los integrantes del triángulo que ocultan el acoso, mientras que en la siguiente hipótesis se tuvo en cuenta la especificidad del rol que ejerce cada estudiante. Los resultados confirman lo hipotetizado, ya que la baja auto-ocultación del bullying posibilita que se tenga un mayor bienestar. En este sentido, los adolescentes que no ocultan el bullying han demostrado tener un bienestar subjetivo mejor de lo que se podría esperar tras el acoso escolar.

La hipótesis 4 no recibió apoyo empírico, ya que, aunque se da un mayor bienestar en el nivel bajo de auto-ocultación para agresores y testigos, no se confirma para el rol de víctima. Este resultado puede ser debido a que las víctimas, como confirmamos en la H1, son las que más auto-ocultan su acoso escolar, y esto contribuye a que no se beneficien del poder que tiene la comunicación de sucesos traumáticos sobre el bienestar (Páez et al., 2011). De ahí, que este estudio abra la puerta a futuras intervenciones sobre su expresión para la reducción del bullying y los problemas asociados al acoso escolar. Muy posiblemente, esta auto ocultación de las víctimas, al estar sufriendo acoso, se relacione con albergar expectativas de que la situación siga igual e incluso empeore, en caso de revelarlo a otros.

Además, se pudo constatar que los estudiantes más jóvenes, que auto-ocultan menos el bullying y presentan una menor frecuencia de acoso, se caracterizan por un mayor bienestar subjetivo; esto aporta evidencia empírica sobre los factores que pueden proteger contra el acoso escolar. En este sentido, las futuras investigaciones deberían corroborar estos resultados a través de estudios longitudinales que permitan analizar estos efectos, evaluando otros indicadores como la exclusión social propuesta por Bustillos et al. (2008); esto sin obviar la importancia de las etapas evolutivas, ya que durante la adolescencia se fomenta la propia autonomía emocional y referencias con los pares/iguales, por lo que la ocultación de problemas tiene un valor adaptativo (Finkenauer et al., 2002).

A través de la H5 se confirmaron los efectos de mediación moderada, tanto para las víctimas como para los agresores, de la auto-ocultación del bullying sobre el bienestar subjetivo tras el acoso escolar. Esta información es convergente con la propuesta teórica de la auto-ocultamiento y bienestar subjetivo de Uysal et al. (2012), así como con los resultados que indican que la ocultación personal de problemas inhibe el bienestar psicológico (Wang et al., 2014; Wismeijer \& Van Assen, 2008).

Por último, sería recomendable replicar estos hallazgos, en otros centros educativos, aplicando tanto las variables estudiadas como otras escalas de medidas implícitas y de observación que posibiliten la detección de otros efectos relevantes. No obstante, esta investigación dentro del contexto escolar permite una caracterización más precisa de bullying, considerando los roles de víctima, agresor y testigo. 


\section{| Conclusiones}

El acoso escolar representa un problema complejo y constituye un campo de estudio en donde quedan aún interrogantes por esclarecer. Es por ello que en este artículo se ha tratado de establecer el camino a través del cual el bullying ejerce su influencia sobre el bienestar subjetivo, concluyendo que es la auto-ocultación del hecho, de forma tal que a menor auto-ocultación mayor bienestar. Además, se ha confirmado cómo el condicionante del rol de víctima, agresor o testigo puede modificar dicho proceso. Los resultados también permiten concluir que el sexo y la edad, junto con el rol, son variables a considerar dentro de la auto-ocultación. Si bien, la auto-ocultación presenta un continuo que va de poco a mucho, en este trabajo y como primera aproximación de estudio se ha planteado la comparación (bajo vs. alto), sin contemplar por ejemplo el punto medio. Es por ello que futuros estudios, y si el número de participantes lo permite, podrían basarse en otras comparaciones a partir de los terciles de la medida.

Se ha constatado que tanto los agresores como las víctimas ocupan puestos clave en la problemática del bullying, sin olvidarse de los ya mencionados testigos, que no se atreven a informar del problema que están observando y consistiendo; hecho que revierte sobre el bienestar subjetivo de todos los actores implicados. Así, el estar inmerso en este ambiente de acoso hace que se pueda aprender que lo más adaptativo es la máxima de "sálvese quien pueda" o si me agreden me callo. Así las cosas, es alentador verificar empíricamente que los más jóvenes que no auto-ocultan el episodio de bullying sobrellevan mejor la situación.

Lo que también se puede afirman, a partir de los conocimientos existentes sobre acoso escolar, es la importancia de trabajar con las víctimas, agresores y testigos, para evitar de este modo más sufrimiento. La justicia restaurativa es un ejemplo de intervención en este sentido, que se propone aquí como una práctica de colaboración conjunta en la escuela; así como las estrategias de intervención basadas en la expresión, vista la influencia que la auto-ocultación tiene sobre el bienestar subjetivo. En definitiva, es fundamental concienciar al conjunto de la sociedad que es básico no mantener en secreto esta práctica, ya que así resulta muy difícil solucionar el problema.

Dada la relevancia que tiene el rol de los estudiantes en el acoso, es oportuno reseñar que las etiquetas en el presente trabajo son elegidas por los participantes, cuando ellos se auto-identifican como víctimas, agresores o testigos. Aunque como se ha constatado, tras la consulta de los trabajos revisados sobre bullying, no se puede hablar de categorías puras, de modo que efectivamente los observadores pueden jugar diferentes roles más o menos activos en el desarrollo del acoso, así como el rol de agresor-víctima que tiende a darse con cierta frecuencia, o la diferencia entre víctimas pasivas y activas. Es por ello que estos hallazgos abren nuevas posibilidades de investigación donde se analice cómo un mismo participante, en función de las circunstancias sociales o evolución de los episodios de acoso, pueda ir modificando su rol.

Finalmente, una cuestión que debería abordarse también en futuros trabajos es el papel que juegan los docentes, padres e instituciones, responsables del bienestar de los discentes, en las relaciones entre pares que se han descrito en este estudio; especificamente cuando el adulto recibe una revelación de bullying por parte de un estudiante, pues de su respuesta e implicación puede depender el cese o agravamiento de las conductas de hostigamiento. Resolución que tiene consecuencias no sólo para la víctima, sino para todos los implicados en el proceso. 


\section{| Referencias}

Bello, G. (2014). Diferencia negativa, alteridad e interhumanidad. En M. N. Quiles del Castillo, J. F. Morales, S. Fernández, y M. D. Morera (Eds.), Psicología de la Maldad. Cómo todos podemos ser Caín (pp. 103-119). Edición Grupo 5.

Bustillos, A., Silván-Ferrero, M. P., Gaviria, E., y Morales, J. F. (2008). Variables psicosociales y acoso escolar: el papel de las normas de grupo y la identificación grupal. Revista de Psicología Social, 23, 151-161. http://dx.doi.org/10.1174/021347408784135779

Conde, S., y Ávila, J. (2018). El maltrato escolar en centros de educación primaria en la provincia de Huelva (España). Psicodebate, 18, 51-71. http://dx.doi.org/10.18682/pd.v18i1.743

Craig, W., Harel-Fisch, Y., Fogel-Grinvald, H., Dostaler, S., Hetland, J., Simons-Morton, B., Molcho, M., Gaspar de Mato, M., Overpeck, M., Due, P., Pickett, W., the HBSC Violence \& Injuries Prevention Focus Group, \& the HBSC Bullying Writing Group (2009). A cross-national profile of bullying and victimization among adolescents in 40 countries. International Journal Public Health, 54, 216-224. http://dx.doi.org/10.1007/s00038-009-5413-9

Cuevas, M. C., y Marmolejo, M. A. (2016). Observadores: un rol determinante en el acoso escolar. Pensamiento Psicológico, 14, 89-102. http://dx.doi.org/10.11144/Javerianacali.PPSI14-1.orda

Darley, J. M. (1996). How organizations socialize individuals into evildoing. En D. M. Messik, \& A. E. Tenbrunsel (Eds.), Codes of Conduct, Behavior Research into business ethics (pp. 13-43). Russel Sage Foundation.

Defensor del Pueblo. (2007). Informes, estudios y documentos. Violencia escolar: Maltrato entre iguales en la Educación Secundaria Obligatoria 1999-2006. Nuevo estudio y actualización del Informe 2000. https://www.defensordelpueblo.es/noticias/acoso-y-violencia-escolar/

Diener, E., Emmons, R., Larsen, R. J., \& Griffin, S. (1985). The satisfaction with life scale. Journal of Personality Assessment, 49, 71-75. https://doi.org/10.1207/s15327752jpa4901_13

Diener, E., Suh, E., Lucas, R., \& Smith, H. (1999). Subjective well-being: Three decades of progress. Psychological Bulletin, 125, 276-302. http://dx.doi.org/10.1037/0033-2909.125.2.276

Finkenauer, C., Engels, R., \& Meeus, W. (2002). Keeping secrets from parents: Advantages and disadvantages of secrecy in adolescence. Journal of Youth and Adolescence, 31, 123-136. https://doi.org/10.1023/A:1014069926507

Fundación Ayuda a Niños y Adolescentes en Riesgo -ANAR- (2018). III Estudio sobre el acoso escolar y ciberbullying según los afectados. https://www.anar.org/wp-content/uploads/2018/09/III-Estudio-sobre-acoso-escolar-y-ciberbullying-seg\%C3\%BAn-los-afectados.pdf

Garaigordobil, M., Martínez-Valderrey, V., y Machimbarrena, J.M. (2017). Intervención en el bullying y cyberbullying: Evaluación del caso Martín. Revista de Psicología Clínica con Niños y Adolescentes, 1, 25-32.

Hayes, A. F. (2009). Beyond Baron and Kenny: statistical mediation analysis in the new millennium. Communication Monographs, 76, 408-420. http://dx.doi.org/10.1080/03637750903310360

Hayes, A. F. (2018). Introduction to mediation, moderation and conditional process analysis. A regression-based approach (2 Ed.). The Guilford Press.

Heintzelman, S. J., \& Diener, E. (2018). Subjective well-being, social interpretation, and relationship thriving. Journal of Research in Personality, 78, 93-105. http://dx.doi.org/10.1016/j.jrp.2018.11.007

Instituto Nacional de Estadística -INE-. (2019). Acoso escolar, datos, cifras y estadísticas. https://www.epdata.es/datos/acoso-escolar-datos-cifras-estadisticas/257/espana/106 
Klocke, A., Clair, A., \& Bradshaw, J. R. (2014). International variations in subjective well-being. Child Indicators Research, 7, 1-20. http://dx.doi.org/10.1007/s12187-013-9213-7

Larson, D. G., Chastain R. L., Hoyt W. T., \& Ayzenberg R. (2015). Self-concealment: Integrative review and working model. Journal of Social and Clinical Psychology, 34, 705-774. http://dx.doi.org/10.1521/jscp.2015.34.8.705

Larson, D. G., \& Chastain R. L. (1990). Self-Concealment: Conceptualization. Measurement, and Health Implications. Journal of Social and Clinical Psychology 9, 439-455. http://dx.doi.org/10.1521/jscp.1990.9.4.439

Lenci, M., \& Matuga, J. (2010). The lifetime bully: Investigating the relationship between adolescent bullying and depression in early adulthood. Journal of School Counseling, 8, 7-7.

Ministerio de Educación, Cultura y Deporte -MECD-. (2017). Informe Anual. Servicio de atención telefónica de casos de malos tratos y acoso en el ámbito de los centros docentes del sistema educativo español. https://sede.educacion.gob.es/publiventa/descarga.action?f_codigo_agc $=18856$

Menesini, E., \& Salmivalli, C. (2017). Bullying in schools: the state of knowledge and effective interventions. Psychology, Health and Medicine, 22, 240-253. http://dx.doi.org/10.1080/13548506.2017.1279740

Millán Reyes, L. C., Barrera Sanchez, L. F., y Ospina Diaz, J. M. (2015). Caracterización del bullying en estudiantes de medicina de Tunja, Boyacá. Revista Virtual Universidad Católica del Norte, (45), 101-112. http://revistavirtual.ucn.edu.co/index.php/RevistaUCN/article/view/659/1191

Navarro, R., Ruiz-Oliva, R., Larrañaga, E., \& Yubero, S. (2015). The impact of cyberbullying and social bullying on optimism, global and school-related happiness and life satisfaction among 10-12-year-old schoolchildren. Applied Research in Quality of Life, 10, 15-36. https://doi.or$\mathrm{g} / 10.1007 / \mathrm{s} 11482-013-9292-0$

Olweus, D. (1993). Bullying at school: What we know and what we can do. Blackwell.

Olweus, D. (2003). A profile of bullying at school. Educational Leadership, 60, 12-17.

Olweus, D. (2011). Bullying at school and later criminality: findings from three Swedish community samples of males. Criminal Behaviour and Mental Health, 21, 151-156. https://doi.org/10.1002/cbm.806

Olweus, D. (2013). School bullying: Development and some important challenges. Annual Review of Clinical Psychology, 9, 751-780. https://doi.org/10.1146/annurev-clinpsy-050212-1855\&16

Oñate, A. y Piñuel, ı. (2010). Informe Cisneros X: Acoso y Violencia Escolar en España. https://www.bienestaryproteccioninfantil.es/imagenes/tablaContenidos03SubSec/acoso-escolar_cisneros.pdf

Páez, D., Martin Beristaín, C., Gonzalez, J. L., Basabe, N., y de Rivera, J. (2011). Superando la Violencia Colectiva y Construyendo Cultura de Paz. Fundamentos.

Peets, K., Pöyhönen, V., Juvonen, J., \& Salmivalli, C. (2015). Classroom norms of bullying alter the degree to which children defend in response to their affective empathy and power. Developmental Psychology, 51, 913-920. http://dx.doi.org/10.1037/a0039287

Puértolas, A., y Montiel, I. (2017). Bullying en educación secundaria: una revisión sobre las características de las víctimas y las víctimas-acosadoras y las consecuencias de su victimización. Revista de Victimología, 5, 85-128.

Quiles del Castillo, M. N., Morales, J. F., Fernández, S., y Morera, M. D (2014). Psicología de la Maldad. Cómo todos podemos ser Caín. Edición Grupo 5.

Rettew, D. C., \& Pawlowski, S. (2016). Bullying. Child and Adolescent Psychiatric Clinics of North America, 25(2), 235-242. http://dx.doi.org/10.1016/j.chc.2015.12.002 
Saarento, S., \& Salmivalli, C. (2015). The Role of Classroom Peer Ecology and Bystanders Responses in Bullying. Child Development Perspectives, 9(4), 201-205. http://dx.doi.org/10.1111/cdep. 12140

Salmivalli, C. (2013). El acoso y el grupo de iguales. En A. Ovejero, P. K. Smith, y S. Yubero (Eds.). El acoso escolar y su prevención. Perspectivas internacionales (pp. 111-130). Biblioteca Nueva.

Uysal, A., Lin, H. L., Knee, C. R., \& Bush, A. L. (2012). The association between self-concealment from one's partner and relationship well-being. Personality and Social Psychology Bulletin, 38(1), 39-51. https://doi.org/10.1177/0146167211429331

Varela, J. J., Savahl, S., Adams, S., \& Reyes, F. (2020). Examining the Relationship Among Bullying, School Climate and Adolescent Well-Being in Chile and South Africa: a Cross Cultural Comparison. Child Indicators Research, 13, 819-838. https://doi.or$\mathrm{g} / 10.1007 / \mathrm{s} 12187-019-09648-0$

Wang, J., Qi, L., \& Cui, L. (2014). The mediating effect of personality traits on the relationship between self-concealment and subjective well-being. Social Behavior and Personality, 2(4), 695-704. http://dx.doi.org/10.2224/sbp.2014.42.4.695

Wismeijer, A., \& Van Assen, M. (2008). Do neuroticism and extraversion explain the negative association between self-concealment and subjective well-being? Personality and Individual Differences, 45, 345-349. http://doi.org/bgdpj9

Zhou, S., King, E. J., Gjorgiovska, J., Mihajlov, A., \& Stojanovski, K. (2019) Self-concealment, discrimination, and mental health in Macedonia: Disparities experienced by sexual and gender minorities. Global Public Health, 14. 1075-1086. https://doi.or$\mathrm{g} / 10.1080 / 17441692.2018 .1560484$ 